\title{
Another hurrah for a 'smoke-free' environment
}

"Tobacco surely was designed to poison and destroy mankind."

Philip Morin Freneau

American poet, 1790

Was it my imagination, or did I really hear a cheer throughout this country on Feb 25, 1990 , when the smoking ban went into effect on all domestic airline flights in the continental United States? And, do I not hear another collective cheer for the Department of Health and Human Services Secretary's vigorous and steadfast campaign against tobacco companies and their advertising practices?

As I wrote in my editorial in the December 1987 issue of JAOA, "Despite the tobacco companies' slick promotion of smoking and their labored attempts to rebut and deny the evidence of tobacco's deadly effects, few Americans now believe that smoking is anything other than what the Surgeon General called 'the chief preventable cause of death in our society." "

And, was not King James I of England prophetic when, in 1604, he said, "A custom loathsome, hateful to the nose, harmful to the brain, dangerous to the lungs, and in the black, stinking fume thereof, the nearest resembling the horrible Stygian smoke of the pit that is bottomless"?

In previous editorials, I have called on the healthcare profession to take the lead in the effort to ban smoking. Osteopathic medical school and hospital administrators have done a splendid job in establishing programs to create smoke-free environments. They have found that a program that includes a time frame for compliance, employee reimbursement for smoking cessation classes, and recognition awards for the employees' personal health promotion and disease prevention efforts encourages smokers in their efforts to give up the habit.

Now, there is something else to cheer about. Mr John Perrin, Executive Director of the American Osteopathic Association, is formulating plans to make the AOA Central office smoke-free. We are concerned about the difficulties that some of the few AOA employee smokers may experience. Therefore, we will encourage their enrollment in smoking cessation programs by reimbursing them for registration fees in these programs. We will also present a health promotion/disease prevention recognition award to those employees who successfully "kick the habit."

We can all be proud of the efforts at the AOA Central office, and we congratulate all of those who have worked so hard on behalf of health promotion. It's another-but definitely not the last-hurrah for a smoke-free environment.
THOMAS WESLEY ALLEN, DO Editor in Chief 

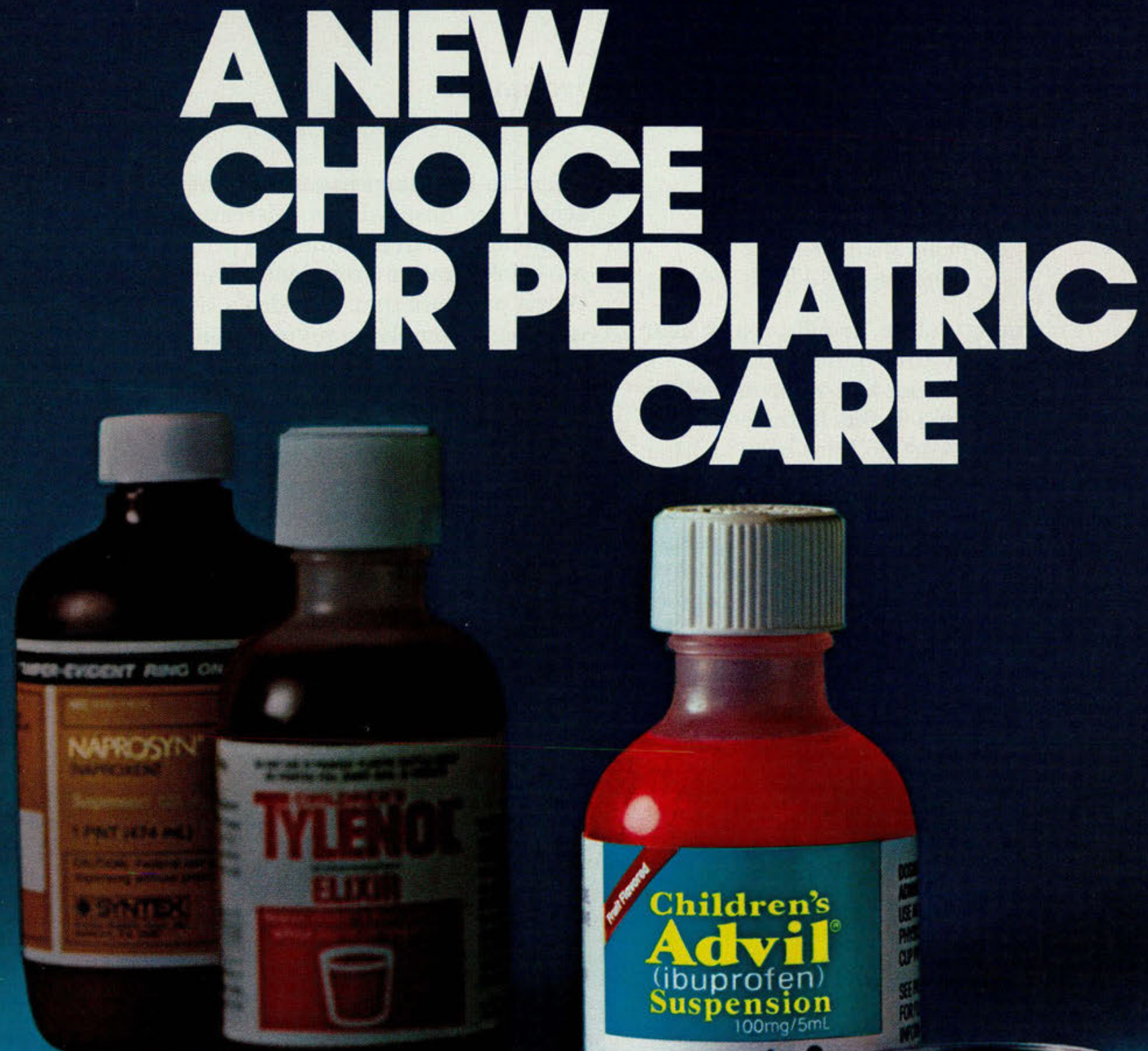


\section{EFFECTIVELY REDUCES FEVER}

Clinical trials have documented the significant antipyretic efficacy of Children's Advil ${ }^{\circledR}$ (ibuprofen) Suspension. ${ }^{1,2}$

\section{EFFECTIVELY REDUCES INFLAMMATION IN JUVENILE ARTHRITIS \\ Children's Advil (ibuprofen) Suspension} produced significant improvement in all efficacy parameters-including number of swollen and painful joints, grip strength, and duration of morning stiffness. ${ }^{3}$

\section{WELL TOLERATED}

at clinically effective doses ranging from 20 to $50 \mathrm{mg} / \mathrm{kg} / \mathrm{day}$

\section{BY PRESCRIPTION ONLY}

Please see following page for a brief summary of prescribing information.
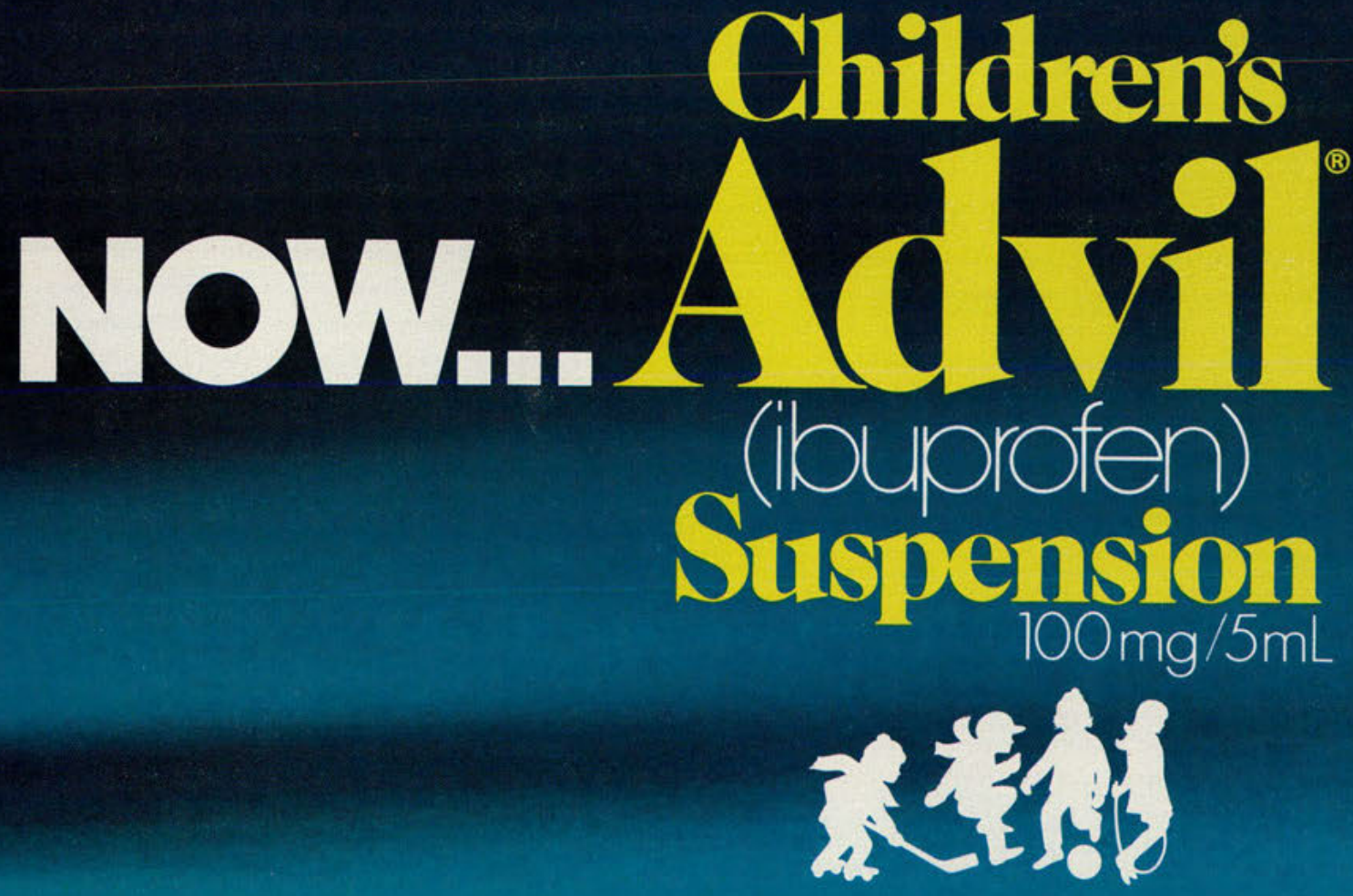


\section{Children's \\ A NEW CHOICE
FOR PEDIATRIC CARE}
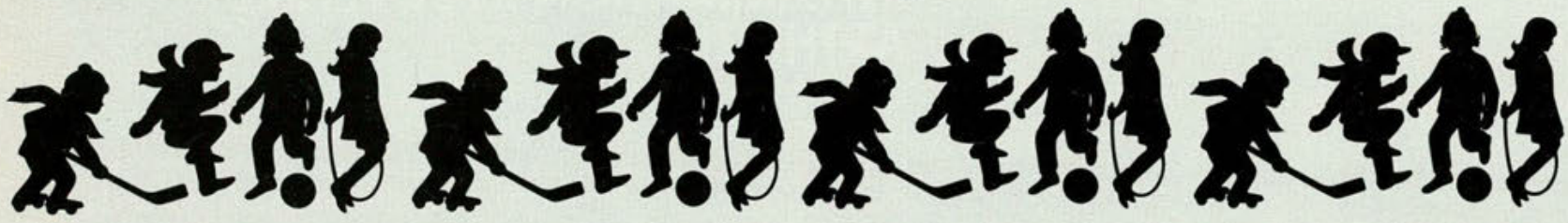

BRIEF SUMMARY OF PRESCRIBING INFORMATION

CONTRAINDICAMONS: Patlents hypersensitive to buprofen or patients with all or part of the syndrome of nasal polyps, angioedema and bronchospastic reactivity to aspirin or other nonsteroidal anti-inflammatory agents. Anaphylactoid reactions to ibuprofen have occurred in such patients. WARNINGS: Risk of Gi Ulcerations, Bleeding, and Perforation with NSAD Therapy: Physicians should remain alert for ulceration and bleeding in patients treated chronically with NSADS even in the absence of previous GI tract symptoms. In patients observed in clinical trials of several months' to two years duration, symptomatic upper Gl ulcers, gross bleeding or perforation appear to occur
in approximately $1 \%$ of patients treated for $3-6$ months, and in about $2.4 \%$ of patients treated for one year.

Except for a prior history of serious $G 1$ events and other risk foctors known to be associated with pep tic ulcer disease, no risk factors have been associated with increased risk Elderly or debilitated patients seem to tolerate ulceration or bleeding less w

PRECAUTONS: Generat: Because serious GI troct ulceration and bleeding can occur without warning symptoms, physicians should follow chronically treated patients for the signs and symp-

toms of ulceration and bleeding a patient develops such complaints the drug should be discontinued and the patient should have

an ophthalmologic examination. with caution in patients with a history of cardiac decompensation or hypentension.

SUSPENSION should be used with caution in persons with intrinsic coagulation detects and those on anticoagulant therapy.

The antipyrefic and anti-inflammatory activity of ibuproten may reduce fever and inflammation thus diminishing their utility as diagnostic signs in defecting complications of presumed noninfectious, noninflammatory painful conditions.

Small decreases (usually not exceeding one gram) in hernoglobin and hematocrit with an apparent dose response relationship have been observed following chronic administration. If there are no signs of bleeding it is probably not clinically importan.

To avoid exacerbation of disease or adrenal insufficiency, patients on prolonged corticosteroid therapy should have their therapy tapered slowly when CHILDRENS ADVL * SUSPENSION is added to the treatment program

meningitis with fever and coma has been observed on rare occasions therapy. Although it is more likely to occur in patients with systemic and related connective fissue diseases, it has been reported in adult patien Who do not have underlying chronic disease. If signs or symptoms of meningitis develop in a
patient on CHILDREN'S ADVIL SUSPENSION the possibility of its being related to ibuprofen should be

Renal Effects: As with other nonsteroidal anti-inflammatory drugs, long-term administration of ibuprofen to animals has resulted in renal papiliary necrosis and other abnormal renal pathology. In humans, there have been reports of acute interstitial nephritis with hematuria. proteinuria, and occasionally nephrotic syndrome.

A second form of renal toxicity has been seen in patients with prerenal conditions leading to reducthon in renal blood how or bloodvolume. In these patients, administration of a nonsteroidal antlcipitate impoired renal function heart fallure liver dystunction and those toking diuretics and the elderty. Those patients at high risk who chronically take CHILDREN S ADVLL SUSPENSION should have rena function monitored if they have signs or symptoms of azotemia. Discontinuation of nonsteroidal anti-inflammatory drug therapy is typically followed by recovery to the pretreatment state. Since ibuprofen is eliminated primarily by the kidneys. patients with significantly impaired renal function should be closely monitored and a reduction in dosage should be anticipated to avoid

Information for Patlents: Physicians may wish to discuss with their patients the potential risks and likely benefits of treatment with CHILDRENSADML * SUSPENSION

Labordiory Tests. Meaningtul (3 imes the upper imit of normal) elevations of SGPT or SGOT (AST)

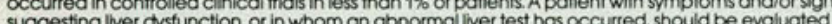
for evidence of the development of more severe hepatic reactions while on therapy with ibupro fen. If abnormal liver tests persist or worsen if clinical sians and symptoms consistent with liver disease develop, or if systemic manifestations occur, CHILDRENS ADVL * SUSPENSION should be discontinued.

Dlabetes: Each $5 \mathrm{~mL}$ of CHIILRENSSADVL * SUSPENSION contains $2.5 \mathrm{~g}$ of sucrose which should be taken into consideration when freating patients with impaired glucose tolerance. It also contains $350 \mathrm{mg}$ of sorbitol per $5 \mathrm{~mL}$. Although in clinical trials CHILDRENSADVL * SUSPENSION was not associated with more diarrhea than control treatments, should a potient develop diarrhea, the physician may wish to review the patients dietary intake of sorbitol from other sources

Drug Interactions: Coumarin-type Anficoagulants: Bleeding has been reported when ibuprofen and olher nonsteroldal anti-Intammalory agents hove been administered to patients on cou

Marin-type anticoagulants, the physician should

Aspirin: Concurrent use of aspirin is not recommended.

Methotrexate: In vitro studies indicate that ibuprofen could enhance the toxicity of methotrexate. Caution should be used if CHILDRENS ADML * SUSPENSION is administered concomitantly with methotrexate.

$\mathrm{H}_{2}$ Antagonists: in studies with human volunteers, coodministration of cimetidine or ranitidine with ibuprofen had no substantive effect on ibuprofen serum concentrations.

Furosemide: lbuproten can reduce the natriuretic effect of furosemide and thiazides in some patients. During concomitant therapy with CHILRENS ADVI: SUSPENSION, the patient should be observed closely for signs of renal failure os well as to assure diuretic efficacy. Uthium: lbuprofen produced an elevation of plasma lithium levels $(15 \%)$ and a reduction in renc lithium clearance $(19 \%)$ in a study of 11 normal volunteers during the period of concomitant drug administration. Patlents should be observed carefully for signs of lithium toxicity. Read package insert for lithium before its use.

Pregnancy: Administration of ibuprofen is not recommended during pregnancy or for use by nursing molners.

\section{months have not been established.} ADVL* SUSPENSION is gastrointestinal. In clinical trials among odults involving chronic administration of ibuprofen the percentage of patients reporting one or more gastrointestinal complaints ranged from $4 \%$ to $16 \%$.

Incidence Greater Than 1\% (but less than 3\%), Probable Causal Relationship Gastrointestinar: Nausea," epigastric poin, hearnburn diarthea, abdominal distress, nousea and vomiting indigestion, constipation, abdominal cramps or pain fulliness of the Gl tract (bloating and flatulence)

Central Nervous System: Dizziness" headache, nervousness.

Dermatologic: Rosh (including maculopapular typel: pruritus

Special Senses: Tinnifus.

.

(generally responds promptly to drug discontinuation)

(see PRECAUTONS)

(but less than 1\%), Probable Causal Relationship

Gasirointestinat: Gastric or duodenal ulcer with bleeding and/or pertoration, gastrointestinal hemorthoge, pancreatifis, melena, gastritis, hepatitis, jaundice, abnormal iver function tests. Dermatologic: Vesiculobullous eruptions, urticaria, erythema multiforme, Stevens-Johnson syndrome, alopecia

Central Nervous System: Depression, insomnia, confusion emotional lability, somnolence, aseptic meningitis with fever and come

specia/ Senses: Hearing loss, amblyopia (blurred and/or diminished vision, scotomata and/or

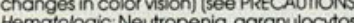

oplastic anemia, hemolytic anemia (sometimes hemoglobin and hematocrit (see PRECAUTIONS).

Cardiovascular: Congestive heart foilure in patients with marginal cardiac function elevated blood pressure, polpitations

Allergic: Syndrome of abdominal pain fever, chills, nausea and vomiting anaphylaxis, bron.

Renat: Acute renal failure in patients with preexisting significantly impaired renal function (see PRECAUTIONS), decreased creatinine clearance, polyuria geme Miscellaneous: Dry eyes and mouth gingival ulcers, thinitis

(1\%) Causal Relationship Unknown

Central Nervous System: Paresthesias, hallucinations, dream abnormalities, pseudotumor cerebri.

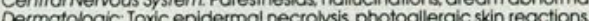

Special Senses Coniunctivitis, diplopia, optic neuritis, cataracts.

Hematologic: Bleeding episodes (eq epistaxis, menorrhogia).

Metaboliciendocrine: Gynecomastia,

Cardiovascular: Arthythmias (sinus tachycardia, sinus bradycardia).

OVERDOSAGE: Patients with a history of ingestion of greater than $100 \mathrm{mg} / \mathrm{kg}$ should have induced emesis or gastric lavage. Multiple dose oral administration of activated charcoal may be usefu. Supportive therapy may include oxygen, respiratory support, and parenteral fluids. Beccuse the drug is acidic and excreted in the urine, administration of sodium
diuresis may be beneficial. DOSAGE AND A.MiNisinanON: Juvenie arthritis: $30-40 \mathrm{mg} / \mathrm{kg}$ day in 3 or 4 divided doses greater than $102.5^{\circ} \mathrm{F}$ every $6-8$ hours (children); $400 \mathrm{mg}$ every 4.6 hours (adults) Rater than 102.5200 mg per day in 3 or 4 divided doses
Mild to moderate pain in adults: $400 \mathrm{mg}$ every 4 to 6 hours

Dysmenorrhed: $400 \mathrm{mg}$ every 4 hours
HOW SUPPUED: 4 and 16 oz bottles

Caution: Federal low prohibits dispensing without prescription.

References: ment of febrile children. Clin Pharmacol Ther. 1989:46:9-17.

Independent Clinical Study. Reduction of Fever in Children. Multiple Dose. Data on file, Medica

3. Independent Clinical Study. Juvenile Arthritis. Data on file, Medical Department, Whitehall 3. Independent
Laboratories.

WHITEHALL LABORATORIES

A HEALTH CARE DMISION OF

AMERICAN HOME PRODUCTS CORPORATION

(c) 1989 Whitehall Laboratories, N.Y, N.Y. 


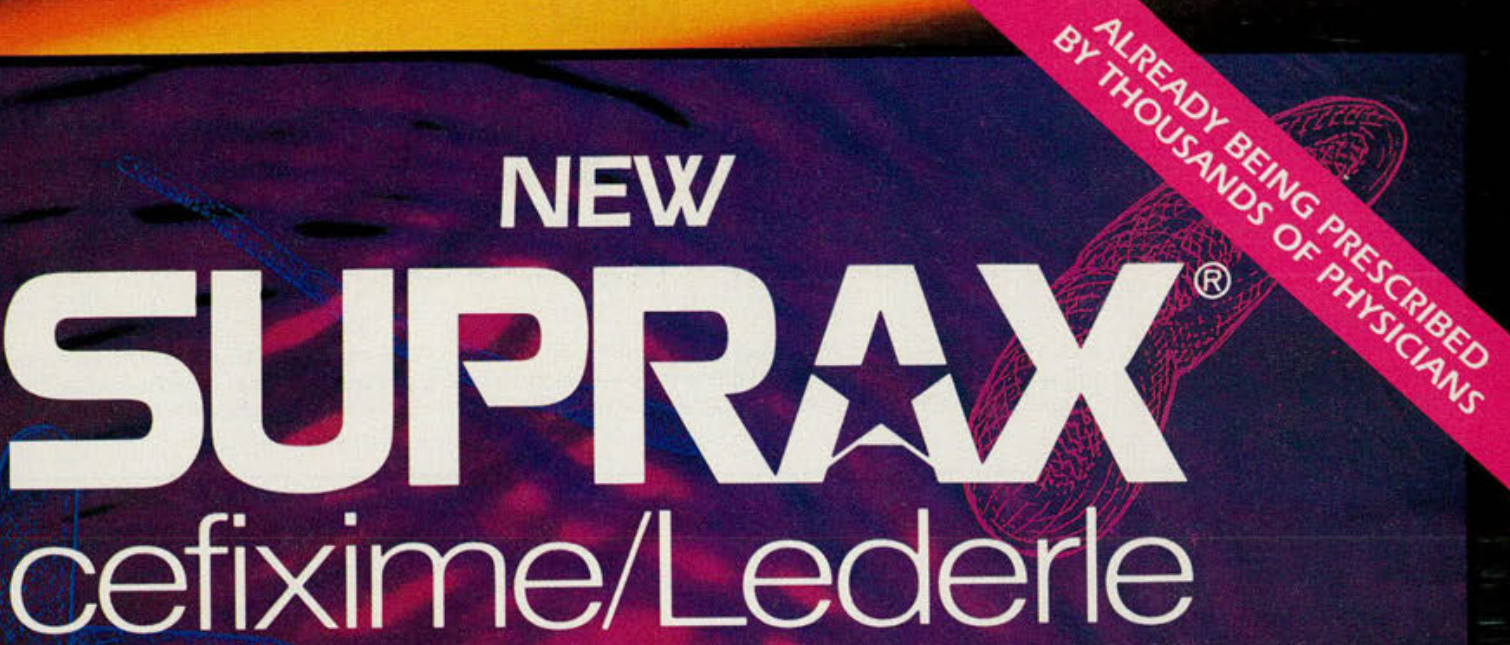

\section{ORAL THIRD GENERATION CEPHALOSPORIN}

Qnce-a-Day therapy for the Treatment of:

- Otitis media Acute bronchitis

- Acute exacerbations of chronic bronchitis

- Pharyngitis $\cdot$ Tonsillitis • UTI

Potent In Vitro Activity Against Major Pathogens Isolatedin Respiratory Jract Infections

Outstanding $\beta$-Lactamase Stability ${ }^{1-3}$

Effective qd or bid Regardless of Severity of Infection 


\section{NEW}

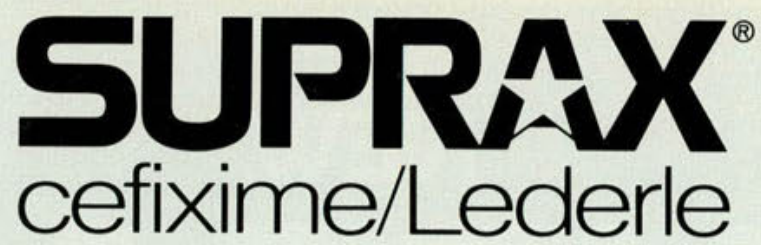

REFERENCES:

1. Sanders CC: $\beta$-Lactamase stability and in vitro activity of oral cephalosporins against strains possessing well-characterized mechanisms of resistance. Antimicrob Agents Chemother 1989:33:1313-1317. 2. Neu HC: In vitro activity of a new broad spectrum, beta-lactamase-stable oral cephalosporin, cefixime. Pediatr Infect Dis J 1987:6:958-962.

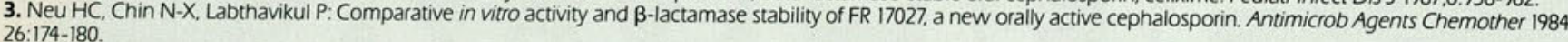

\section{SUPRAX* cefixime/Lederle}

BRIEF SUMMARY. Please see package insert for full Prescribing Information

NDICATIONS AND USAGE

Otitis Media caused by Haemophilus influenzae (beta-lactamase positive and negative strains. Moraxella (Branhamella) catarrhalis (most of which are beta-lactamase positive), and Streptococcis pyogenes."

Note: For information on otitis media caused by Streprococcus pneumonize, see CLINICAL STUDIES section

Acute Bronchitis and Acute Exacerbations of Chronic Bronchitis caused by 5 pneumoniae and $H$ fare positive and negative strains

Perform culture and susceptibility studies to determine causative organism and its susceptibility to SUPRAX. Therapy may begin while waiting for study results and may be adjusted when results are

\section{Pharyngitis and Tonsillitis caused by Spyogenes.}

Note: Penicillin is the usual drug of choice in the treatment of Spyogenes infections, including the prophylaxis of rheumatic fever. SUPRAX is generally effective in the eradication of S pyogenes from the nasopharynx, however, data establishing the efficacy of SUPRAX in the subsequent prevention of rheumatic fever are not available.

Uncomplicated Urinary Tract Infections caused by Escherichia coli and Proteus mirabilis

\section{CLINICAL STUDIES}

In clinical trials of otitis media in nearly 400 children between the ages of 6 months and 10 years. 5 pneumoniae was isolated from $47 \%$ of the patients, $H$ influenzae from $34 \%, B$ catarrhalis from , and $S$ pyogenes from $4 \%$

The overall response rate of $S$ pneumoniae to cefixime was approximately $10 \%$ lower and that of $H$ infivenzae or $B$ catarrhalis approximately $7 \%$ higher $112 \%$ when beta-lactamase positive strains of $H$ influenzae are included) than the response rates of these organisms to the active control drugs. In these studies, patients were randomized and treated with either cefixime at dose regimens $4 \mathrm{mg} / \mathrm{kg}$ bid or $8 \mathrm{mg} / \mathrm{kg}$ qd, or with a standard antibiotic regimen. Sixty-nine to $70 \%$ of the patients in each group had resolution of signs and symptoms of otitis media when evaluated two to four weeks posttreatment, but persistent effusion was found in $15 \%$ of the patients. When evaluated at the completion of therapy, $17 \%$ of patients receiving cefixime and $14 \%$ of patients receiving effective comparative drugs $/ 18 \%$ including those patients who had $H$ influenzae resistant to the control drug and who received the control antibiotic) were considered to be treatment failures. By the two- to four-week follow-up. a total of $30 \%$ to $31 \%$ of patients had evidence of either treatment failure or recurrent disease.

Bacteriological Outcome of Otitis Media at Two- to Four-Weeks Posttherapy Based on Repeat Middle Ear Fluid Culture or Extrapolation from Clinical Outcome

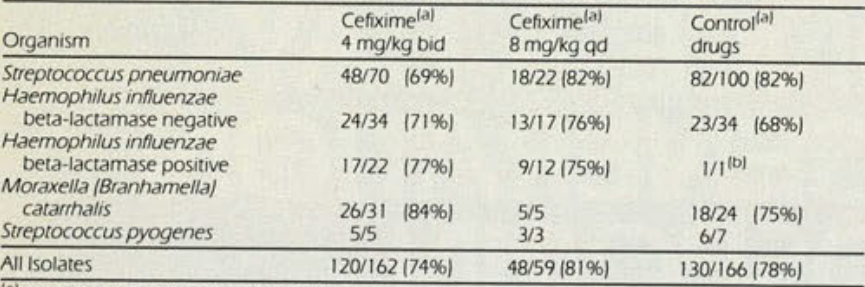

a) Number eradicated/number isolated

(b) An additional 20 beta-lactamase positive strains of $H$ influenzae were isolated, but were excluded from this analysis because they were resistant to the control antibiotic. In 19 of these he clinical course could be assessed, and a favorable outcome occurred in 10. When these cases are included in the overall bacteriological evaluation of therapy with the control drugs, $40 / 185(76 \%)$ of pathogens were considered to be eradicated

Tablets should not be substituted for suspension when treating otitis media

CONTRAINDICATIONS

Known allergy to cephalosporins.

WARNINGS

BEFORE THERAPY WITH SUPRAX IS INSTITUTED, CAREFUL INOUIRY SHOULD BE MADE TO DETERMINE WHETHER THE PATIENT HAS HAD PREVIOUS HYPERSENSITIVITY REACTIONS TO CEPHALOSPORINS, PENICILLINS, OR OTHER DRUGS. IF THIS PRODUCT IS TO BE GIVEN TO PENICILLIN-SENSITIVE PATIENTS, CAUTION SHOULD BE EXERCISED BE BE GIVEN BECAUSE CROSS-HYPERSENSITIVITY AMONG BETA-LACTAM ANTIBIOTICS HAS BEEN
CLEARLY DOCUMENTED AND MAY OCCUR IN UP TO $10 \%$ OF PATIENTS WITH A HISTORY OF PENICILLIN ALLERGY. IF AN ALLERGIC REACTION TO SUPRAX OCCURS, DISCON TINUE THE DRUG. SERIOUS, ACUTE HYPERSENSITIVITY REACTIONS MAY REOUIRE TREATMENT WITH EPINEPHRINE AND OTHER EMERGENCY MEASURES, INCLUDING OXYGEN, INTRAVENOUS FLUIDS, INTRAVENOUSANTIHISTAMINES, CORTICOSTEROIDS, PRESSOR AMINES, AND AIRUVAY MANAGEMENT, AS CLINICALLY INDICATED.

Administer cautiously to allergic patients.

Treatment with broad-spectrum antibiotics alters the normal flora of the colon and may permit overgrowth of clostridia. Studies indicate that a toxin produced by Clostridium difficile is a primary cause of severe antibiotic-associated diarmea including pseudomembranous colitis. Pseudomembranous colitis has been reported with the use of SUPRAX and other broad-spectrum antibiotics lincluding macrolides, semisynthetic penicillins, and cephalosporins). It is important to consider this diagnosis in patients who develop diarmea in association with antibiotic use. Symptoms of pseudomembranous colitis may occur during or after antibiotic treatment and may range in severity from mild to life threatening. Mild cases usually respond to drug discontinuation alone. Moderate-tosevere cases should be managed with fluid, electrolyte, and protein supplementation. When the colitis is not relieved by drug discontinuance, or when it is severe, oral vancomycin is the drug of choice for antibiotic-associated pseudomembranous colitis produced by $C$ difficile Other causes of colitis should be excluded.

\section{PRECAUTIONS}

General: Prolonged use may result in overgrowth of nonsusceptible organisms. If superinfection occurs, take appropriate measures.

Carefully monitor patients on dialysis. Adjust dosage of SUPRAX (cefixime) in patients with renal impairment and those undergoing continuous ambulatory peritoneal dialysis and hemodialysis DOSAGE AND ADMINISTRATION

Prescribe cautiously in patients with a history of gastrointestinal disease, particularly colitis. Drug Interactions: No significant drug interactions have been reported to date. Drug/Laboratory Test Interactions: A false-positive reaction for ketones in the urine may occur with tests using nitroprusside but not with those using nitroferricyanide

SUPRAX administration may result in a false-positive reaction for glucose in the urine using Clinitest ${ }^{* \cdots}$, Benedict's solution, or Fehling's solution. Use glucose tests based on enzymatic glucose oxidase reactions (such as Clinistix $* * *$ or Tes-Tape $* * *$ )

A false-positive direct Coombs test has been reported during treatment with other cephalosporin antibiotics: therefore, it should be recognized that a positive Coombs test may be due to the drug Carcinogenesis, Mutagenesis, Impairment of Fertility: Although no lifetime animal studies have been conducted to evaluate carcinogenic potential, no mutagenic potential of SUPRAX was found in standard laboratory tests. Reproductive studies revealed no fertility impairment in rats at found in standard laboratory tests. Reproductive

Usage in Pregnancy: Pregnancy Category B: Reproduction studies have been performed in mice Usage in Pregnancy: Pregnancy Category $B$ : Reproduction studies have been performed in mice
and rats at doses up to 400 times the human dose and have revealed no evidence of harm to the fetus due to SUPRAX

There are no adequate and well-controlled studies in pregnant women. Because animal reproduction studies are not always predictive of human response, this drug should be used during pregnancy only if clearly needed

Labor and Delivery: SUPRAX has not been studied for use during labor and delivery. Treatment should only be given if clearly needed

Nursing Mothers: It is not known whether SUPRAX is excreted in human milk. Consider discontinuing nursing temporarily during treatment with this drug.

Pediatric Use: Safety and effectiveness of SUPRAX in children aged less than 6 months have not

The incidence of gastrointestinal adverse reactions, including diarthea and loose stools, in pedatric patients receiving the suspension, was comparable to adult patients receiving tablets ADVERSE REACTIONS

Most adverse reactions observed in clinical trials were of a mild and transient nature. Less than four percent (4\%) of patients in the US trials discontinued therapy because of drug-related adverse reactions. Commonly seen adverse reactions in US trials of the tablet formulation were gastrointestinal events, which were reported in $30 \%$ of adult patients on either the bid or the qd regimen. Clinically mild gastrointestinal side effects occurred in $20 \%$ of all patients, moderate events occurred in $9 \%$ of all patients, and severe adverse reactions occurred in $2 \%$ of all patients. Individual event rates included diarthea $16 \%$, loose or frequent stools $6 \%$, abdominal pain $3 \%$, nausea $7 \%$, dyspepsia $3 \%$, and flatulence $3 \%$. The incidence of gastrointestinal adverse reactions, including diarmea and loose stools, in pediatric patients receiving the suspension was comparable to adult patients receiving

Symptoms usually responded to symptomatic therapy or ceased when SUPRAX was

discontinued.

Several patients developed severe diarmea and/or documented pseudomembranous colitis, and a few required hospitalization.

The following adverse reactions have been reported following the use of SUPRAX. Incidence rates were less than 1 in 50 (less than $2 \%$ ), except as noted above for gastrointestinal events. Gastrointestinal: Diarrhea, loose stools, abdominal pain, dyspepsia, nausea, and vomiting. Several cases of documented pseudomembranous colitis were identified during the studies. The onset of pseudomembranous colitis symptoms may occur during or after therapy Hypersensitivity Reactions: Skin rashes, urticaria, drug fever, and pruritus.

Hepatic: Transient elevations in SGPT, SGOT, and alkaline phosphatase.

Renal: Transient elevations in BUN or creatinine

Central Nervous System: Headaches $3 \%$; dizziness.

Hemic and Lymphatic Systems: Transient thrombocytopenia, leukopenia, and eosinophilia. Prolongation in prothrombin time was seen rarely.

Other: Genital pruritus, vaginitis, candidiasis.

The following adverse reactions and altered laboratory tests have been reported for cephalosporin-class antibiotics:

Adverse Reactions: Allergic reactions including anaphylaxis, Stevens-Johnson syndrome, erythema multiforme, toxic epidermal necrolysis, superinfection, renal dysfunction, toxic nephropathy. hepatic dysfunction, including cholestasis, aplastic anemia, hemolytic anemia, hemorrhage. Several cephalosporins have been implicated in triggering seizures, particularly in patients with renal impairment when the dosage was not reduced (see DOSAGE AND ADMINISTRATION and OVERDOSAGE). If seizures associated with drug therapy occur, discontinue drug. Administer anticonvulsant therapy if clinically indicated.

Abnormal Laboratory Tests: Positive direct Coombs test, elevated bilirubin, elevated LDH, pancytopenia, neutropenia, agranulocytosis.

\section{OVERDOSAGE}

Gastric lavage may be indicated: otherwise, no specific antidote exists. Cefixime is not removed in significant quantities from the circulation by hemodialysis or peritoneal dialysis. Adverse reactions in small numbers of healthy adult volunteers receiving single doses up to $2 \mathrm{~g}$ of SUPRAX did not differ from the profile seen in patients treated at the recommended doses.

"Clinitest and Clinistix are registered trademarks of Ames Division, Miles Laboratories, Inc. Tes-Tape is a registered trademark of Eli Lilly and Company. 


\section{THEFIGHAGANSTCANCER
ALSOCAIS FORANAYE BALL.}

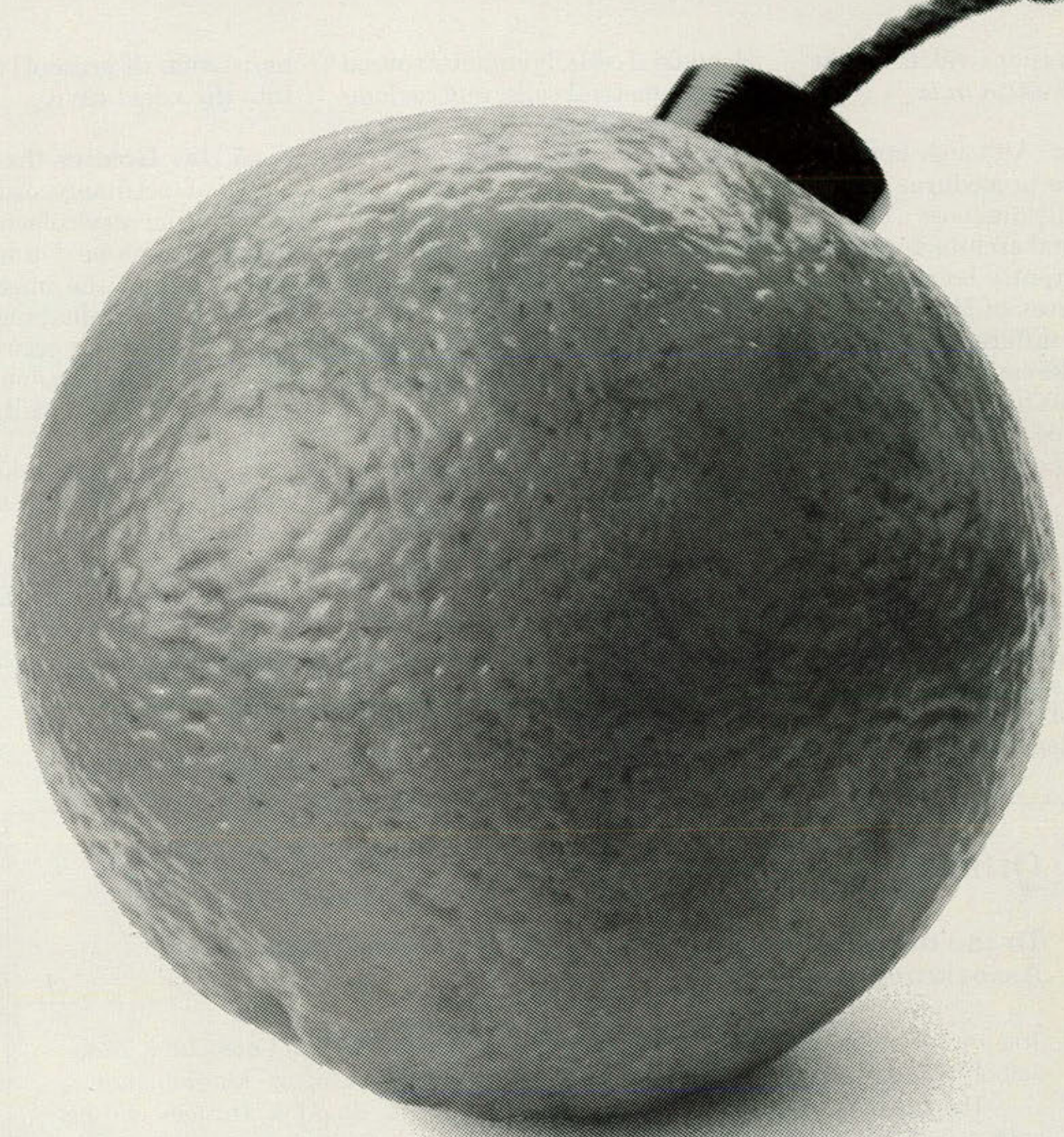

Prepare yourself forVictory at $\mathrm{C}$. Because a diet that includes oranges, peppers and other foods high in Vitamin $C$ may lessen your risk in the battle against cancer. So who else are your allies? Try fiber-rich foods, lowfat dairy products, cruciferous vegetables like cauliflower, poultry, fish, and foods high in Vitamin A. They make up the menu in The Great American Food Fight Against Cancer - an American Cancer Society-sponsored program to lessen your cancer risk. You can learn more by calling 1-800-ACS-2345, or your local American Cancer Society. Before long, you'll $\mathrm{C}$ things our way. 